\title{
Design of a machine for the universal non-contact measurement of large free-form optics with $30 \mathrm{~nm}$ uncertainty
}

\author{
Rens Henselmans ${ }^{*}$, Nick Rosielle ${ }^{1}$, Maarten Steinbuch ${ }^{1}$, Ian Saunders ${ }^{2}$, Rob Bergmans ${ }^{3}$ \\ ${ }^{1}$ Technische Universiteit Eindhoven, Department of Mechanical Engineering, Den Dolech 2, \\ 5600 MB Eindhoven, The Netherlands \\ ${ }^{2}$ TNO Science and Industry, Opto-mechanical Instrumentation, Precision Mechanics department, \\ Stieltjesweg 1, 2628 CK Delft, The Netherlands \\ ${ }^{3}$ NMi VSL B.V., Section Length, Thijsseweg 11, 2629 JA Delft, The Netherlands
}

\begin{abstract}
A new universal non-contact measurement machine design for measuring free-form optics with $30 \mathrm{~nm}$ expanded uncertainty is presented. In the cylindrical machine concept, an optical probe with $5 \mathrm{~mm}$ range is positioned over the surface by a motion system. Due to a $2^{\text {nd }}$ order error effect when measuring smoothly curved surfaces, only 6 position measurement errors are critical (nanometer level). A separate metrology system directly measures these critical errors of the probe and the product relative to a metrology frame, circumventing most stage errors.

An uncertainty estimation has been performed for the presented design, including a calibration uncertainty estimation and a dynamic analysis. Machine dynamics certainly cause relative motion between probe and product, but due to the non-contact nature of the measurement and the short metrology loop, these motions do not cause significant measurement errors. The resulting shape measurement error for aspheres up to medium free-forms is between 24 and 37 $\mathrm{nm}$, and $30-85 \mathrm{~nm}$ for medium to heavily free-form surfaces. The suitability of the proposed design is herewith confirmed. A detailed design and a prototype of the machine are currently being developed.
\end{abstract}

Keywords: Non-contact, universal, large, free-form, aspherical, optics, measurement, machine, probe, metrology

\section{INTRODUCTION}

Aspherical and free-form optics offer great advantages over conventional flat and spherical surfaces. Currently, slow/fast tool diamond turning ${ }^{1}$ and local deterministic polishing techniques ${ }^{2}$ are enabling generation of these complex surfaces (Figure 1.1). The lack of a suitable metrology tool for verification of the surface form is however preventing the widespread application of these advanced optics. In a joint project by TNO, TU/e, NMi VSL and NPL called NANOMEFOS (Nanometer Accuracy NOn-contact MEasurement of Free-form Optical Surfaces), a new measurement machine is being developed. This machine is designed for universal and non-contact measurement of free-from surfaces with $30 \mathrm{~nm}$ expanded measurement uncertainty.

Classical testing of flats and spheres is done with imaging interferometric techniques ${ }^{3}$. By applying surface specific null lenses or computer generated holograms ${ }^{4}$, aspheres and free-forms can be measured, but not universally. By sub-aperture stitching ${ }^{5}$, surfaces with relatively small local departures from spherical

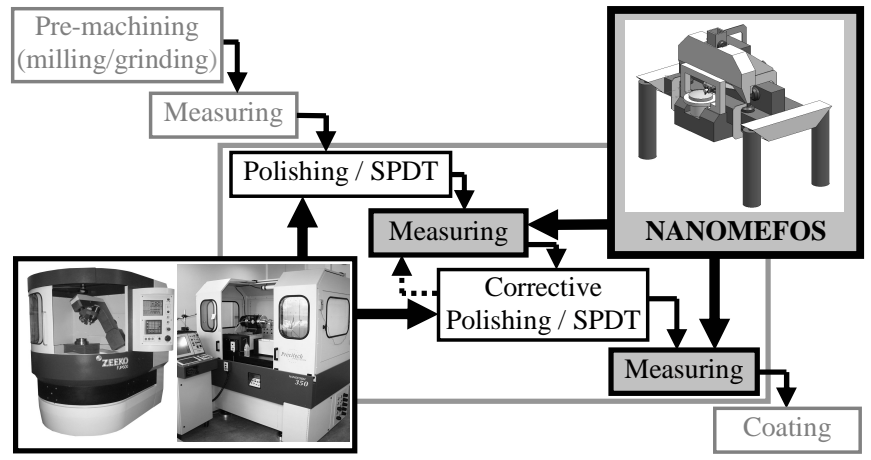

Figure 1.1: Free-form optics manufacturing value chain

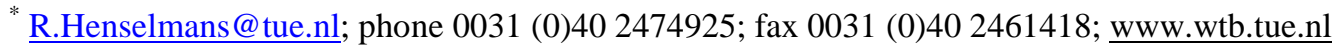


may be measured universally. When local curvature and slope increase, the aperture size has to be reduced, which increases the risk of stitching errors. In contrast to imaging methods, scanning methods allow for universal measurement of all surface types. Coordinate measuring machines with high accuracy have been developed ${ }^{6,7,8}$, but none is yet able to measure steeply sloped surfaces non-contact. Swing arm profilometers ${ }^{9}$ may measure non-contact with a suitable optical probe, but with limited universality. By numerically integrating optically measured slope ${ }^{10,11}$ or curvature or $^{12}$ measurements, aspheres and free-forms (except discontinuous surfaces) can be measured non-contact with high resolution. The absolute form accuracy of the measurements is however limited by numerical integration errors.

After a survey of current measurement methods, a high accuracy scanning coordinate measuring machine design with an optical probe was considered to be most viable for non-contact universally measuring complex optical surfaces with high accuracy. The design and uncertainty estimation of this machine is presented in this paper. After explaining the requirements, the concept will be presented. Next, the machine design, consisting of a non-contact probe, a motion system and a metrology system will be shown. Finally, the shape measurement uncertainty will be estimated by a calibration and dynamical analysis.

\section{REQUIREMENTS}

\subsection{Universal form measurement of flat, spherical, aspherical, free-form and off-axis surfaces}

High-end optics production usually is a typical single-piece production environment. Therefore the metrology system is designed for universally measuring flat, spherical, aspherical, free-form and off-axis surfaces. The surfaces may be convex as well as concave with slopes up to + or $-45^{\circ}$. The maximum product dimensions have been chosen to be $\varnothing 500$ $\mathrm{mm} \times 100 \mathrm{~mm}$, weighing up to $50 \mathrm{~kg}$.

For (rotationally symmetric) aspheres no principal boundary for the departure from the best-fit-sphere is assumed. In practice however, this departure will probably be in the order of millimeters. Based on an estimate of future needs for (non-rotationally symmetric) free-forms, a maximum peak-to-valley departure of $5 \mathrm{~mm}$ from the rotationally symmetric best-fit is assumed. Local slope variations with respect to the rotationally symmetric best fit are estimated to be up to $5^{\circ}$. The surfaces may also be discontinuous (multiple optical surfaces in one part), or have a non-circular circumference.

Reflection as well as transmission optics will be measured, therefore reflectivity will vary between 5 and $100 \%$ and all optical materials (metals, glass, ceramics) may occur. Surface roughness will vary between a few microns and a few nanometers, depending on the stage of manufacturing of the product. When measuring a finished product, it may have multiple layers of coating that will probably influence most optical probing measurement methods. Measuring coated products is therefore left out of consideration.

\subsection{Non-contact}

To prevent polished surfaces from being damaged, the system should measure non-contact. On the one hand this obviously increases the system complexity, but it may also provide an opportunity to greatly increase the measurement speed compared to conventional tactile scanning systems, such as CMMs and profilometers.

\section{3. $30 \mathrm{~nm}$ expanded measurement uncertainty}

Desired form accuracy for high-end optics is up to $\lambda / 20$ for visible light. Therefore the aim is to achieve an expanded measurement uncertainty of $30 \mathrm{~nm}$. This uncertainty is defined as a shell spaced $30 \mathrm{~nm}$ from the real surface, which contains $95 \%$ of all the measurement points (Figure 2.1). As stated in paragraph 2.1, there is great diversity in the products to be measured regarding the form complexity and the roughness. A task specific measurement uncertainty will therefore be calculated, to estimate for which surfaces the $30 \mathrm{~nm}$ is actually achievable.

The measurement should be traceable to the standard of length. No physical free-form standards have however yet been developed for the intended surface shapes and dimensions. Because a universal machine is being designed that can also measure flat and spherical surfaces, traceability may at least be investigated by the use of physical standards for these types. Traceability for aspheres and free-forms will have to be investigated by thorough analysis of the instrument. 


\subsection{Other requirements}

Because the measurement machine is intended to only measure form (not waviness and roughness), a lateral resolution of about $0.5-2 \mathrm{~mm}$ (depending on the complexity and size of the surface) is sufficient. For double-sided optics (transmission optics), usually one side is completely finished first before the other side is manufactured. Therefore only one side needs to be measured.

To keep the measurement practically useful in the workshop and to limit the influence of environmental disturbances, the goal is to achieve a measurement time of about 15 minutes for a $\varnothing 500 \mathrm{~mm}$ surface (acclimatization time not included). The machine should be placed in a conditioned environment, where the temperature is estimated to be $20 \pm$ $0,5^{\circ} \mathrm{C}$. Within the machines enclosure, temperature stability during measurements is estimated to be $\pm 0,2^{\circ} \mathrm{C}$.

\section{CONCEPTUAL DESIGN}

Since the surfaces are always more or less rotationally symmetric, or an off-axis part of such a surface, a cylindrical coordinate measuring machine setup has been designed (Figure 3.1) which scans a non-contact probe over the surface to be measured ${ }^{13}$. Because of the large range of surface slopes to be measured $\left(-45^{\circ}\right.$ to $\left.+45^{\circ}\right)$, the optical distance probe will be oriented perpendicular to the rotationally symmetric best-fit of the surface. The probe is therefore mounted on a rotation axis $\psi$, which is mounted on translation stages $\mathrm{R}$ and $\mathrm{Z}$. This motion system will position the probe with respect to the free-form surface which is continuously rotating on a spindle $\theta$ at about $1 \mathrm{rev} / \mathrm{s}$.

When measuring a free-form product, the surface will depart from rotational symmetry, for instance between the continuous and dotted line in Figure 3.1. To avoid dynamically following the surface with the large $\mathrm{R}$ and $\mathrm{Z}$ stages, the optical probe should have an axial range of $5 \mathrm{~mm}$. This way a free-form surface can be scanned swiftly with minimal dynamics in the system because the $\mathrm{R}$ and $\mathrm{Z}$ stages and the $\psi$-axis can be stationary. It also allows for a track to be measured multiple times for averaging with little effort. Measuring a $500 \mathrm{~mm}$ diameter product with $2 \mathrm{~mm}$ track spacing, 5 revolutions per track at a spindle speed of $1 \mathrm{rev} / \mathrm{s}$ will for instance take about 15 minutes, which is a greatly reduced measurement time compared to conventional tactile systems.

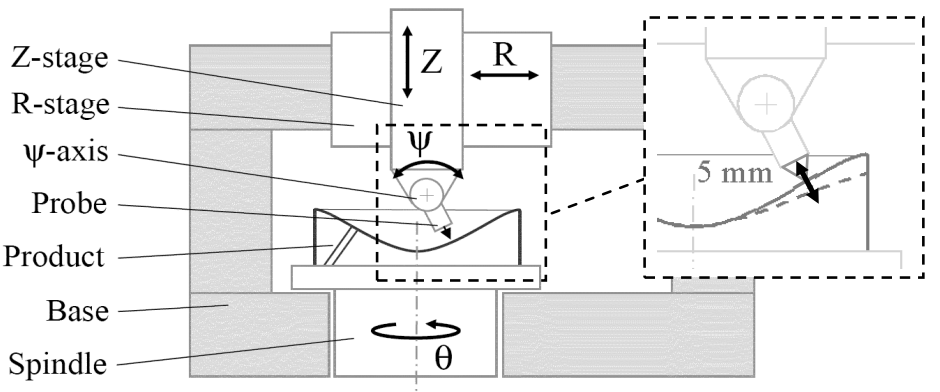

Figure 3.1: Machine concept

The machine will further incorporate an intermediate body on which a product is to be mounted. This intermediate body reproducibly interfaces to the spindle of the measurement machine as well as to other manufacturing machine spindles, to provide quick measurement of a product without the need for re-alignment on the machines.

The uncertainty of the measurement is determined by the uncertainty by which the position of the probe tip relative to the product is known. The dotted metrology loop of Figure 3.2 is long and for instance includes all the errors of the stages and their stability (similar to a conventional CMM). To shorten this loop, a metrology frame has been added from which the probe and product position are measured as directly as possible. The measurement systems will continuously measure relative motion of the probe and the product, which allows for off-line compensation of static and dynamic errors.

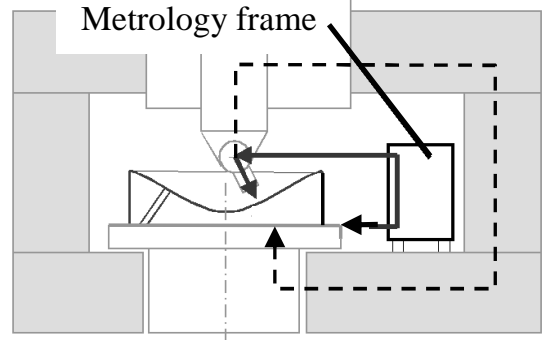

Figure 3.2: Metrology frame

The shape measurement error $(\delta)$ of the machine is the result of 13 potential position measurement errors $(\Delta)$, being 6 degrees of freedom of the product, 6 of the probe and the distance measured by the probe. The surfaces to be measured are supposed to be smooth and have a minimal (local) radius of curvature in the order of tens of millimeters. This implies that when the probe is perpendicular to the surface, tangential errors result in a $2^{\text {nd }}$ order shape measurement error, as shown in Figure 4.3. 

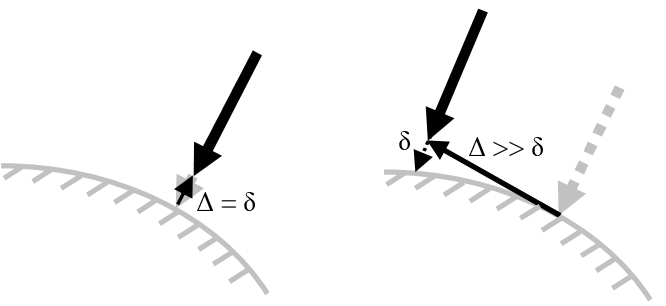

Figure 4.3: $2^{\text {nd }}$ Order measurement error effect

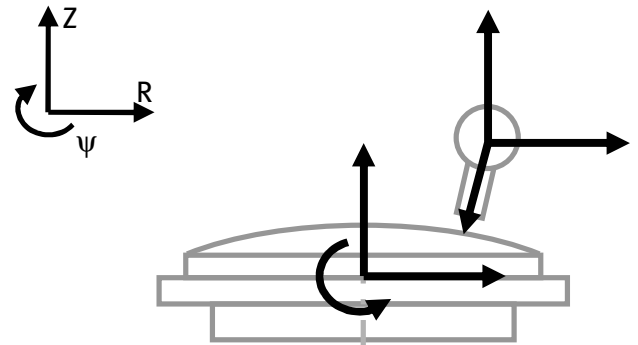

Figure 4.4: Critical error directions

When this effect is taken into account for the expected surface shapes, only 6 of the original 13 position measurement errors appear to be critical (nanometer order), the other 7 are less critical (micrometer order). The 6 critical position measurement errors are the probe $\mathrm{R}$ and $\mathrm{Z}$, the spindle $\mathrm{R}, \mathrm{Z}$ and $\psi$ and the distance measured by the probe (Figure 4.4). The critical directions will have to be measured; the less critical ones could be done mechanically. The critical positioning measurement errors are all within the plane of motion of the probe (the measurement plane), reducing the metrology problem to a $2 \mathrm{D}$ problem. When measuring a free-form, the probe will however not always be perpendicular to the surface, which will be further explained in section 6.3 .

\section{CURRENT MACHINE DESIGN}

Figure 5.1 shows an overview of the current machine design, in which the three main subsystems (the non-contact probe, the motion system and the metrology system) are indicated. In the following paragraphs, each of these will be further discussed. The design of the machine in general and the metrology loop in particular has been patented ${ }^{14}$.

\subsection{Non-contact probe design}

The non-contact probe is a critical and integral part of the machine design. No commercial probe has been found that complies with the requirements described below and therefore a new probe design is currently being developed. The design is however not yet ready for publication. Therefore only the concept will be explained here briefly for completeness of the measurement machine design explanation.

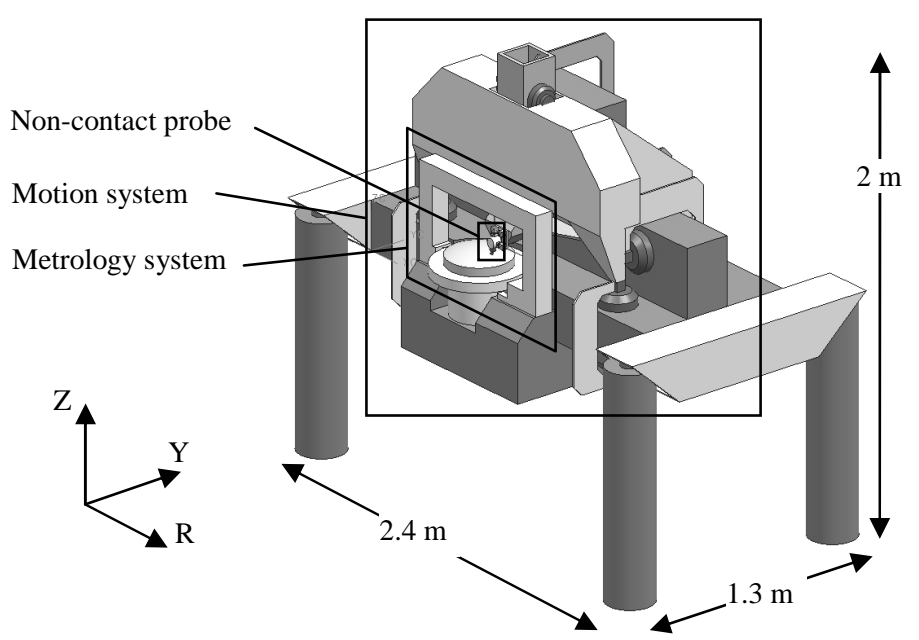

Figure 5.1: Machine design overview

The non-contact probe should measure the distance from the $\psi$-axis to the surface. When measuring heavily free-form surfaces, an axial range of $5 \mathrm{~mm}$ is required. The probe should then also have an angular range of $5^{\circ}$, because it will not be actively servo controlled perpendicular to the free-form surface. When using an optical operating principle, the axial measurement uncertainty will probably be affected by this misalignment. The requirements for the optical probe have therefore been set to an axial measurement uncertainty $(\mathrm{k}=2)$ of $10 \mathrm{~nm}$ when perpendicular, and $35 \mathrm{~nm}$ at $5^{\circ}$ misalignment. The resolution of the probe should be an order of magnitude less, about $1 \mathrm{~nm}$. A point density of $0.5 \mathrm{~mm}$ requires a sample rate of at least $3 \mathrm{kHz}$, but when actively focusing the probe to the surface a higher sample rate will probably be required.

The current concept is a two stage system, consisting of an optical head which measures the absolute distance to the surface over a short range $( \pm 1 \mu \mathrm{m})$, and a long stroke measurement system capable of measuring $5 \mathrm{~mm}$ displacement with nanometer resolution (Figure 5.2). Several optical principles are currently being considered for the optical head, including critical angle autofocus ${ }^{15}$, differential confocal $^{16}$ and absolute interferometry ${ }^{17}$. The long stroke measurement 
system preferably is a laser interferometer or a linear glass scale. The optical head will be mounted onto either an elastic or airbearing linear guidance, and will be servo controlled to maintain focus onto the surface. To have sufficient axial sensitivity with focusing methods, a spot size in the order of $1-2 \mu \mathrm{m}$ will probably be required.

Currently the non-contact probe design is evolving and the requirements still appear feasible. Proof of principle tests will be conducted soon. More details on the optical probe will be presented in future papers.

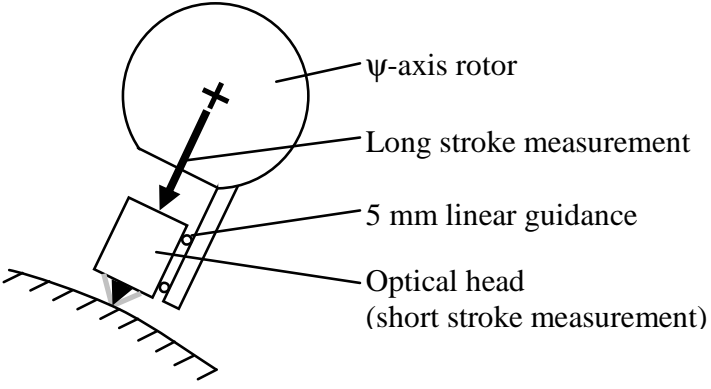

Figure 5.2: Preliminary probe concept

\subsection{Motion system design}

The motion system positions the probe relative to the product. It should provide a stiff and stable structural loop between probe and product and an accurate plane of motion for the probe. The current design of the motion system consists of a granite base, which is built from a vertical and a horizontal plate ( 1 and 2 in Figure 5.3a), and is suspended on air mounts (3). The horizontal plate has a slot in which a BlockHead 10R air-bearing spindle (4) will be mounted. This spindle was chosen for its excellent stiffness and error motion specifications. The spindle will be fitted with a brushless motor and a Heidenhain ERP 880 encoder with 180.000 signal periods. After 200 fold interpolation this will give a resolution of about $45 \mathrm{~nm}$ in tangential direction at a radius of $250 \mathrm{~mm}$.

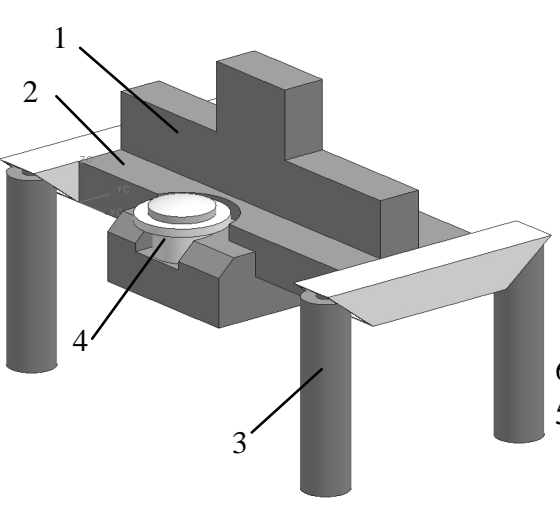

Figure 5.3a: Base and spindle

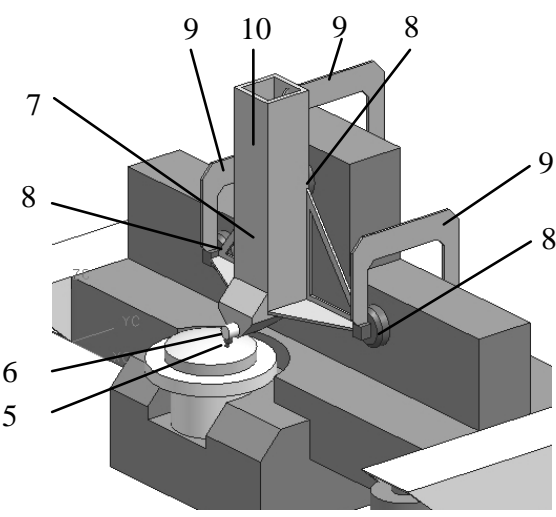

Figure 5.3b: Z-stage

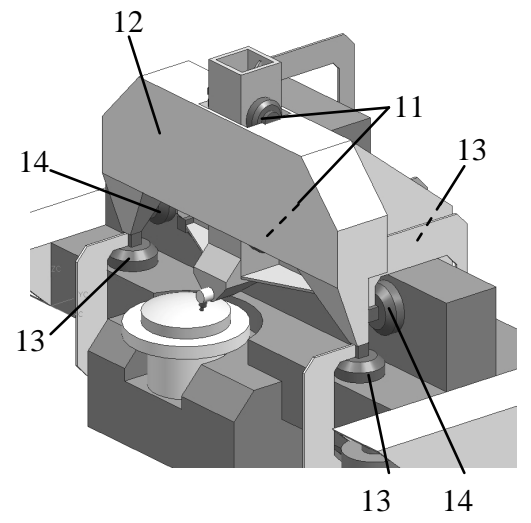

Figure 5.3c: R-stage

The probe (5, Figure 5.3b) will be mounted to the air-bearing $\psi$-axis (6), the housing of which is mounted onto the Zstage (7). The $\psi$-angle of the axis is a less critical error and will be measured with an encoder. Since the vertical plane can be manufactured down to micrometer flatness, the Z-stage will be aligned directly onto this plane with 3 flat $\varnothing 150$ $\mathrm{mm}$ air-bearings (8). This way the inherent flatness and stiffness of the base is transferred to the probe in a short structural loop in y-direction. The air bearings will be pre-loaded with opposite piston mounted air-bearings through the preload frames (9). The Z-stage further contains a ceramic tube (10), the two sides of which will be used to constrain the $\mathrm{R}$ and $\psi$ motion relative to the R-stage via two $\varnothing 150 \mathrm{~mm}$ air bearings (11). A motor and weight compensation are added.

The R-stage (Figure 5.3c) consists of a stiff box (12) that is aligned to the horizontal plate by $3 \varnothing 200 \mathrm{~mm}$ bearings (13). Two $\varnothing 200 \mathrm{~mm}$ bearings (14) constrain the motion in y and $\theta$-direction, leaving only the R-direction free for the stage. Two $\varnothing 150 \mathrm{~mm}$ bearings (11) constrain the R and $\psi$ motion of the Z-stage relative to the R-stage. Since the bearings (14) are aligned to the same vertical plane as bearings (8) of the Z-stage, relative motion between two stages is minimized (apart from $\mathrm{Z}$ of course).

Since the measurement is non-contact, the positioning accuracy of the probe is not very critical (a few $\mu \mathrm{m})$ as long as the metrology system measures the actual position. The position control of the probe can either be done from CAD data, a coarse measurement conducted in advance, or by feedback from the probe. 


\subsection{Metrology system design}

The metrology loop should measure the position of the probe relative to the product within the measurement plane. The probe $\mathrm{R}$ and Z-positions should be measured with a maximum uncertainty of about $15 \mathrm{~nm}$, the product $\mathrm{R}$ and Z-position to about $5 \mathrm{~nm}$ and the tilt to about $0.1 \mu \mathrm{rad}$. Figure 5.4 shows the setup in which the probe position may be measured. There are several configurations possible, with variations in mirror positions etc. but the principle remains the same.

To measure the probe position, a heterodyne interferometer beam (1) is delivered to a non-polarizing beamsplitter (2), for instance with folding mirrors attached to the stages (not shown). From here two beams travel to two polarizing beamsplitters (3). Here, one polarization direction of the beam travels straight through, the other part is deflected to the reference mirror (5). The beam is reflected and passes a $\lambda / 4$ plate (4) for the second time, causing it to pass through the beamsplitter. With a lens (7) the beam is now focused on the center of the reflecting $\psi$-axis rotor (8). After reflection, it passes another $\lambda / 4$ plate (4) for the second time, which now causes it to join the other beam again, resulting in

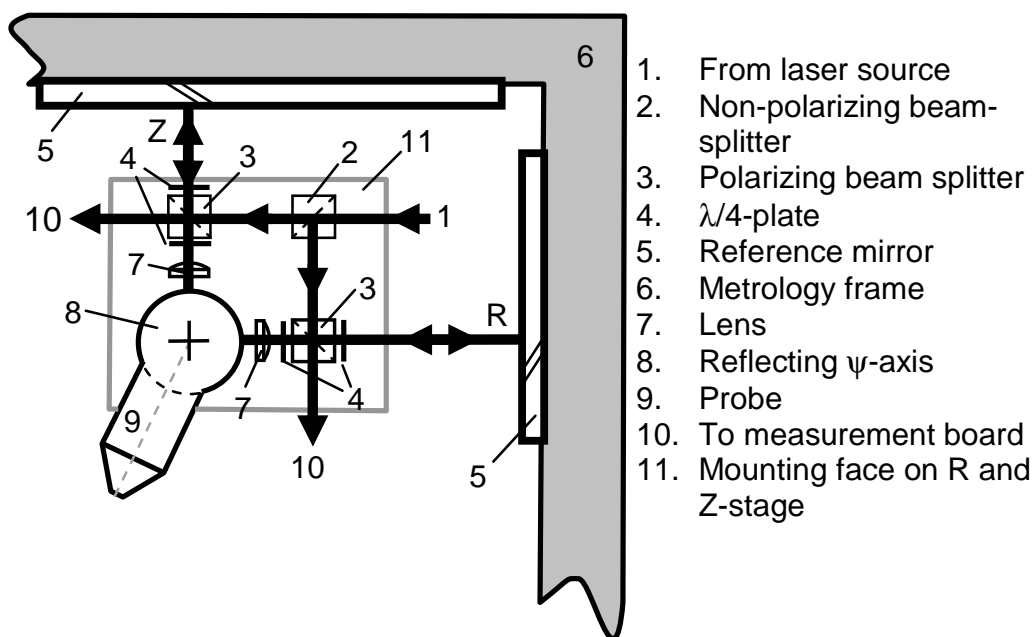

Figure 5.4: Interferometry system concept interference between the two. With mirrors, the beams (10) can now be transported to the receivers.

Basically, one part of the beam travels straight through beamsplitter (3), while the other part makes a detour between the reference mirror and the $\psi$-axis rotor, resulting in OPD between the beams. This way a direct measurement of the probe displacement in $\mathrm{R}$ and Z-direction with respect to the metrology frame has been achieved, free of Abbe errors. All critical stage errors are now measured and can thus be corrected for in the data-processing. As mentioned before, the $\psi$ angle of the probe is a second order error and will be measured with an encoder on the $\psi$-axis.

The product is assumed to be rigidly attached to the spindle rotor. The BlockHead 10R spindle is specified at $<20 \mathrm{~nm}$ axial and radial error motion and $<0.1 \mu \mathrm{rad}$ tilt error motion. The synchronous part of this may be calibrated; the asynchronous part can be measured with capacitive probes measuring to a calibrated edge on the rotor. This way, a short metrology loop between probe and product has been created, in which all the critical positioning errors are measured (Figure 5.5). The metrology frame is shown schematically in this figure; it will be closed at the bottom for stability in future designs.

When measuring a free-form, for instance a toroid of which the surface varies between the continuous and the dotted line of Figure 5.5, the only moving parts in the machine are the continuously rotating spindle and the focusing part of the probe. Since this focusing part may be very light, there will be very little dynamical errors in the system, which will enable high measurement speeds. Most of the vibrations that remain will be measured and can thus be corrected for, which will be further explained in the dynamic analysis of section 6.5 .

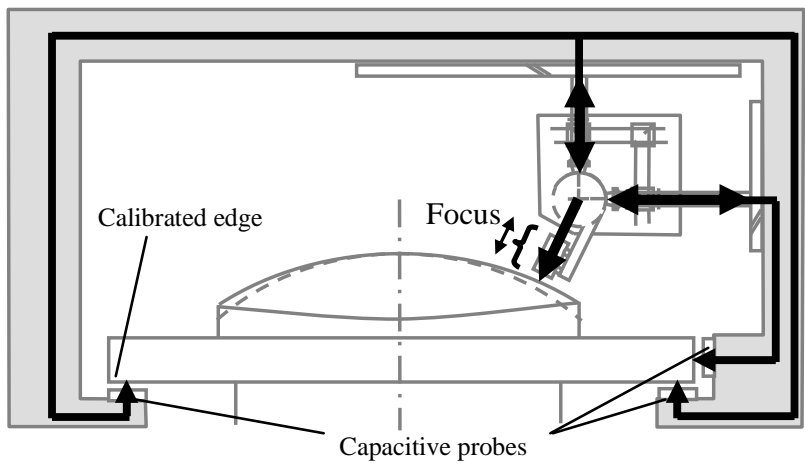

Figure 5.5: Metrology loop

To the above metrology loop design, the beam delivery path and beam shielding from environmental disturbances will be added in the coming period. The spindle measurement method and metrology frame design will also be continued. 


\subsection{Extreme positions}

In Figure 5.6 the extreme positions of the machine are shown, where the parallel guidance of the Z-stage, and the lasers (depicted in black) following the $\psi$-axis can be seen. The probe is shown in top left and bottom right position at - and $+45^{\circ}$. The product is clearly visible and accessible to the operator in this machine setup.

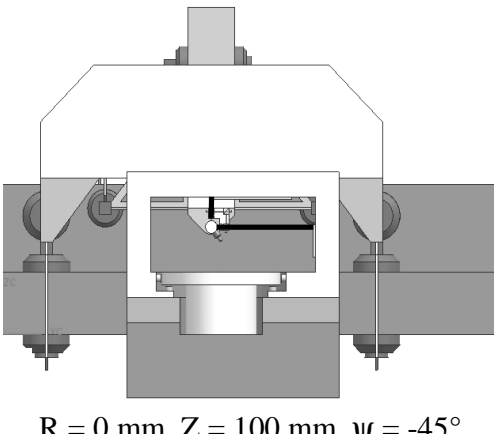

Figure 5.6: Extreme positions

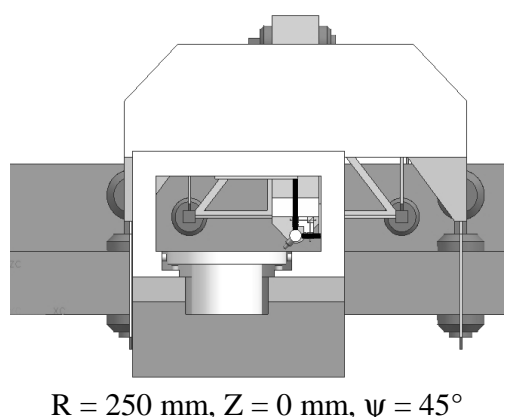

$\mathrm{R}=250 \mathrm{~mm}, \mathrm{Z}=0 \mathrm{~mm}, \psi=45^{\circ}$

\section{SHAPE MEASUREMENT UNCERTAINTY ESTIMATION}

To investigate the potential of the current design, the shape measurement uncertainty has been estimated. Hereto the calibration, thermal, sensor and dynamic uncertainties have been estimated or calculated. These have been summed to obtain the uncertainty vector between the probe tip and the product. This vector, combined with the local surface characteristics, results in a first estimate of the machines task specific measurement uncertainty.

\subsection{Coordinate system}

The machine RYZ-coordinate system is defined in Figure 6.1. The origin ( 0 ) is located at the nominal spindle surface centre position (relative to the base). At the $\psi$-axis mirror centre $\left(0_{\psi}\right)$, a local abc-coordinate system is defined that rotates with the $\psi$ axis. The axes are parallel with the global coordinate system for $\psi=0^{\circ}$. The axial measurement direction of the probe is thus called the c-direction, and local misalignments of the surface to the probe are in $\alpha$ and $\beta$ direction.

\subsection{Surface characteristics}

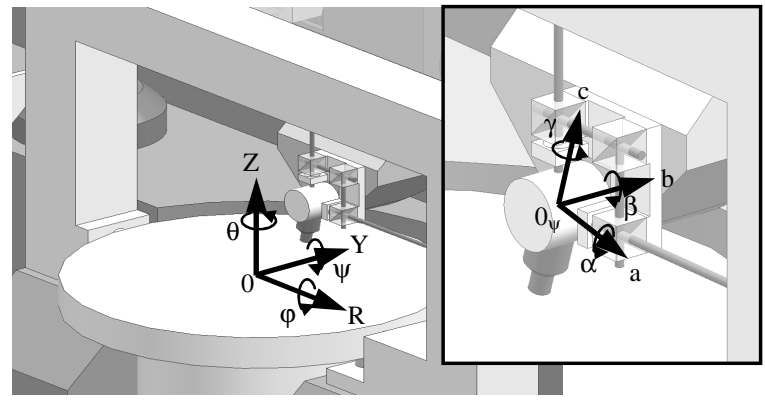

Figure 6.1: Coordinate system

The second order effect of Figure 4.3 holds for rotationally symmetric surfaces (flat, spherical and aspherical), because the probe is then truly perpendicular to the surface (apart from small positioning errors). When measuring a free-form this is however no longer the case (Figure 6.2). As will be shown later, the measurement uncertainty then also depends on the local slope and probe tip acceleration, which is a function of the local curvature and measurement speed. When the smoothly curved surface is approximated with (part) of a sine wave, the maximum slope $(\alpha)$, curvature (d $\alpha / \mathrm{db})$ and required probe tip acceleration $\left(\ddot{c}_{P T}\right)$ are equal to $(6.1)-(6.3)$.

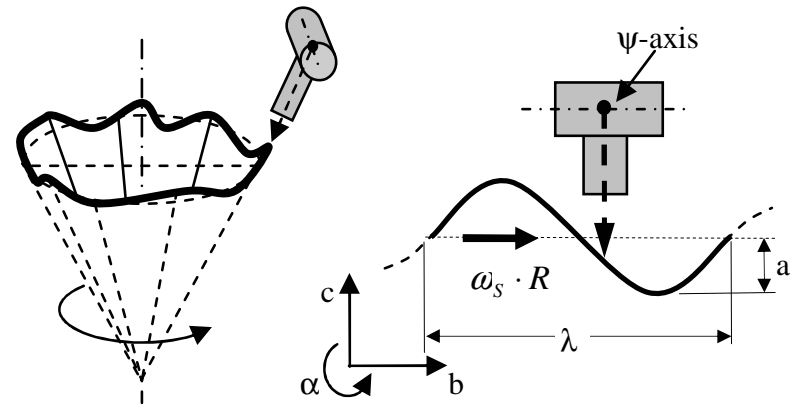

$$
\begin{array}{ll}
\alpha_{\text {max }}=\frac{2 \pi a}{\lambda} & {[\mathrm{rad}]} \\
\left(\frac{d \alpha}{d b}\right)_{\max }=4 \pi^{2} \frac{a}{\lambda^{2}} & {[\mathrm{rad} / \mathrm{m}]} \\
\ddot{c}_{P T, \max }=4 \pi^{2} \frac{a R^{2} \omega_{S}^{2}}{\lambda^{2}}=\left(\frac{d \alpha}{d b}\right)_{\text {max }} R^{2} \omega_{S}^{2} & {\left[\mathrm{~m} / \mathrm{s}^{2}\right]}
\end{array}
$$

Figure 6.2: Free-form slope and curvature

As mentioned before, local slopes up to $5^{\circ}$ are expected. With wavelengths in the order of $0.1 \mathrm{~m}$, curvatures up to 5 $\mathrm{rad} / \mathrm{m}$ can be expected. The dynamic error mainly depends on the reaction force from the required probe tip acceleration, which is a function of the local curvature, radial position and measurement speed as will be further explained in section 6.5 . 


\subsection{Error vector}

The focal point of the probe will be taken as the probe tip, although this is not a physical point. Due to the non-contact nature of the measurement, a positioning error will not directly cause a measurement error as long as the error is measured by the metrology system. Together with the (calibrated) unmeasured directions, the measured probe tip and product position can be calculated, resulting in a measured surface coordinate. Due to measurement errors, this coordinate will differ from the real surface coordinate by error vector $\vec{\Delta}$ (Figure 6.3). The resulting shape measurement error $\delta$ depends on the local surface slope and curvature.

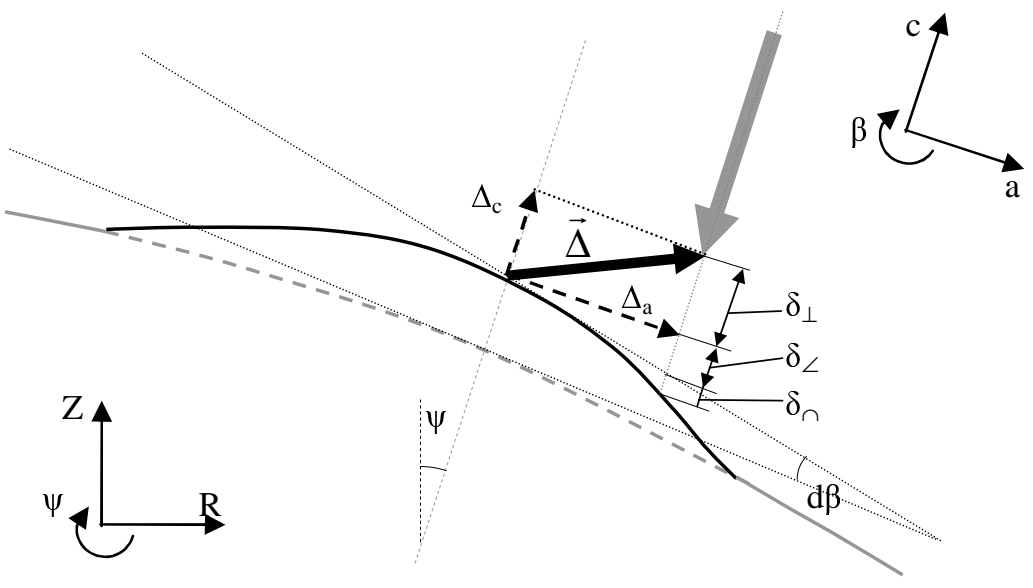

Figure 6.3: Error vector

The error vector can be decomposed into a tangential (a and b-direction) and a perpendicular component (c-direction). An error in c-direction directly results in a distance measurement error $\delta_{\perp}$, a tangential error results in a distance measurement error that depends on the local slope $\left(\delta_{L}\right)$ and curvature $\left(\delta_{\cap}\right)$. Due to the relatively small tangential displacements $\delta_{\cap}$ is negligible.

The tangential error vector $\Delta_{\mathrm{ab}}$ is composed from $\Delta_{\mathrm{a}}$ and $\Delta_{\mathrm{b}}$. The magnitude of $\delta_{\angle}$ depends heavily on the relative direction between $\Delta_{\mathrm{ab}}$ and the resultant direction of the local slope $\angle_{\alpha \beta}$. In worst case the two are parallel, and then $\delta_{\angle}$ is the product of the two. The perpendicular and tangential errors are independent and thus the resulting shape measurement error $\varepsilon$ is equal to:

$$
\varepsilon=\sqrt{\delta_{\perp}^{2}+\left(\delta_{L}+\delta_{\cap}\right)^{2}} \approx \sqrt{\Delta_{c}^{2}+\left(\Delta_{a}^{2}+\Delta_{b}^{2}\right) \cdot \angle_{\alpha \beta}^{2}}
$$

\subsection{Static shape measurement uncertainty}

Calibration, environmental and sensor uncertainties will cause a measurement error that is not dependent of the dynamics of the system. The static uncertainty is the uncertainty with which a single point on a surface can be measured, when all the axes are stationary. Although the calibration procedures still have to be designed, an initial estimate of the expanded calibration uncertainty $U$ has been made (Table 6.1).

\begin{tabular}{|c|c|c|c|c|c|}
\hline \multicolumn{2}{|c|}{ Source } & \multirow{2}{*}{$\begin{array}{l}\mathrm{U}(\mathbf{2} \boldsymbol{\sigma}) \\
5 \mathrm{~nm}\end{array}$} & \multicolumn{2}{|c|}{ Source } & \multirow{2}{*}{$\begin{array}{l}\mathbf{U}(\mathbf{2} \boldsymbol{\sigma}) \\
5 \mu \mathrm{rad}\end{array}$} \\
\hline 1 & Spindle radial error motion & & 12 & $\psi$-axis encoder error & \\
\hline 2 & Spindle axial error motion & $5 \mathrm{~nm}$ & 13 & Probe error & $10 \mathrm{~nm}$ \\
\hline 3 & Spindle tilt error motion & $0.1 \mu \mathrm{rad}$ & 14 & Probe objective guidance & $50 \mathrm{~nm}$ \\
\hline 4 & Spindle encoder error & $2 \mu \mathrm{rad}$ & 15 & Probe obj. guidance alignment & $10 \mu \mathrm{rad}$ \\
\hline 5 & R-reference mirror flatness & $10 \mathrm{~nm}$ & 16 & Probe to surface misalignment & $10 \mathrm{~nm} /{ }^{\circ}$ \\
\hline 6 & R-reference mirror alignment & $0.1 \mu \mathrm{rad}$ & 17 & Vertical base plate flatness & $100 \mathrm{~nm}$ \\
\hline 7 & $\psi$-axis mirror roundness & $10 \mathrm{~nm}$ & 18 & Spindle - vertical base misalignment & $1 \mu \mathrm{rad}$ \\
\hline 8 & R-interferometer error & $5 \mathrm{~nm}$ & 19 & $\psi$-axis axial position & $100 \mathrm{~nm}$ \\
\hline 9 & Z-reference mirror flatness & $10 \mathrm{~nm}$ & 20 & $\psi$-axis perp. to vertical base & $100 \mathrm{~nm} / 90^{\circ}$ \\
\hline 10 & Z-reference mirror alignment & $0.1 \mu \mathrm{rad}$ & 21 & Nulling error R laser & $5 \mathrm{~nm}$ \\
\hline 11 & Z-interferometer error & $3 \mathrm{~nm}$ & 22 & Nulling error Z laser & $5 \mathrm{~nm}$ \\
\hline
\end{tabular}

Table 6.1: Estimated calibration uncertainties 
By implementing self-calibration techniques into the machine design, the uncertainty due to drift between measurements is expected to be reduced to $10 \mathrm{~nm}$ for the metrology frame, $10 \mathrm{~nm}$ for the probe and $250 \mathrm{~nm}$ for the structural loop in $\mathrm{b}$ direction. Further, sensor noise has been estimated by a few times their individual resolution.

Each of the errors has been transformed to the resulting error at the probe tip in abc-direction. The errors are assumed to be independent and normally distributed. The resulting uncertainty vector in $\mathrm{a}, \mathrm{b}$ and c-direction can then be calculated with (6.5).

$$
\vec{U}_{a b c}=\left[\begin{array}{l}
2 \sqrt{\sigma_{a, 1}^{2}+\sigma_{a, 2}^{2}+\ldots+\sigma_{a, n}^{2}} \\
2 \sqrt{\sigma_{b, 1}^{2}+\sigma_{b, 2}^{2}+\ldots+\sigma_{b, n}^{2}} \\
2 \sqrt{\sigma_{c, 1}^{2}+\sigma_{c, 2}^{2}+\ldots+\sigma_{c, n}^{2}}
\end{array}\right]
$$

When the uncertainty vector $\vec{U}_{a b c}$ is implemented as the error vector $\vec{\Delta}$ in 6.4 , the resulting static shape measurement uncertainty as a function of the local slope and measurement position can be calculated. It appears that mainly the radial measurement position significantly influences the measurement uncertainty, and thus the static shape measurement uncertainty can be plotted as a function of the radial measurement position and the local surface slope (Figure 6.4a). The dark area at to bottom indicates the region for which an uncertainty of $30 \mathrm{~nm}$ can be obtained. The uncertainty for rotationally symmetric aspheres (local slope is $0^{\circ}$ ) has also been plotted (Figure 6.4b).

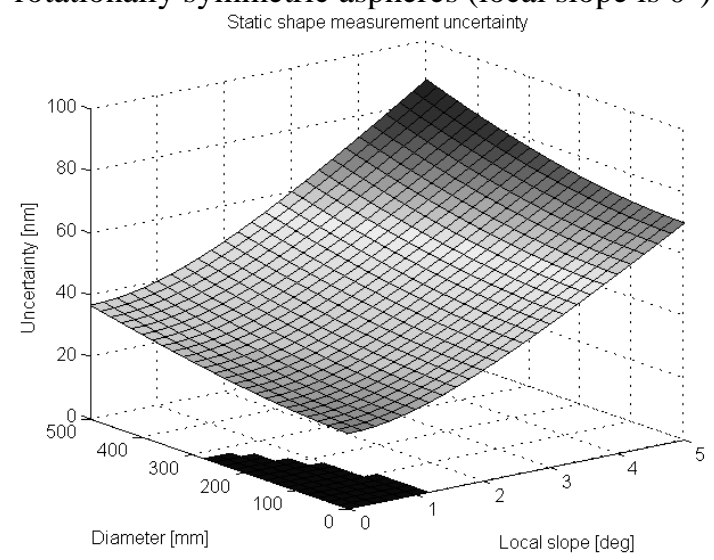

Figure 6.4a: Static shape measurement uncertainty

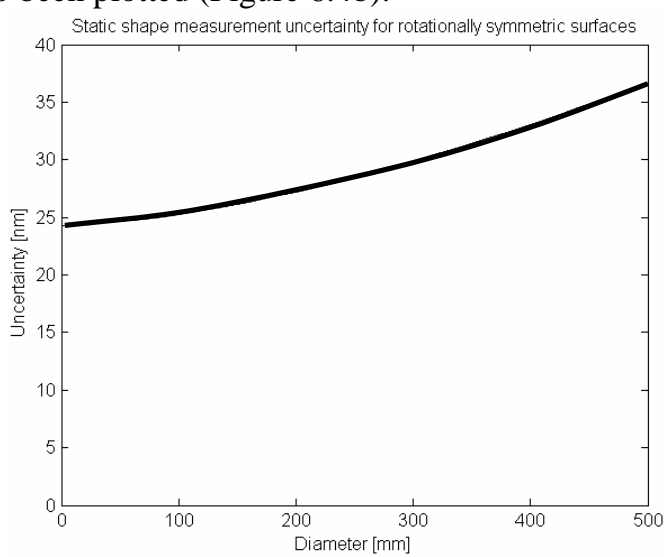

Figure 6.4b: Rotationally symmetric shape measurement uncertainty

\subsection{Dynamic uncertainty}

When measuring a surface, there will be three main sources of vibrations, namely spindle unbalance, probe tip motion and floor vibrations. To investigate the magnitude of these vibrations and their influence on the positioning and measurement errors of the machine a dynamic model has been made in Matlab SimMechanics (Figure 6.5). This 6 DOF model consists of six rigid bodies: base, spindle rotor, R-stage, Z-stage, $\psi$-axis rotor and the probe tip. The main parameters of the model are listed in Table 6.2.

\begin{tabular}{|l|l|}
\hline Parameter & Value \\
\hline Base mass & $2690 \mathrm{~kg}$ \\
\hline Spindle rotor \& product mass & $57-107 \mathrm{~kg}$ \\
\hline R-stage mass & $290 \mathrm{~kg}$ \\
\hline Z-stage mass & $80 \mathrm{~kg}$ \\
\hline$\psi$-axis rotor with probe mass & $1 \mathrm{~kg}$ \\
\hline Moving probe tip mass & $20 \mathrm{~g}$ \\
\hline Bearing $\varnothing 200 \mathrm{~mm}$ stiffness & $7 \cdot 10^{8} \mathrm{~N} / \mathrm{m}$ \\
\hline Bearing $\varnothing 150 \mathrm{~mm}$ stiffness & $3.5 \cdot 10^{8} \mathrm{~N} / \mathrm{m}$ \\
\hline Spindle bearing stiffness & Axial: $1.7 \cdot 10^{9} \mathrm{~N} / \mathrm{m}$ \\
& Radial: $3.5 \cdot 10^{8} \mathrm{~N} / \mathrm{m}$ \\
\hline
\end{tabular}

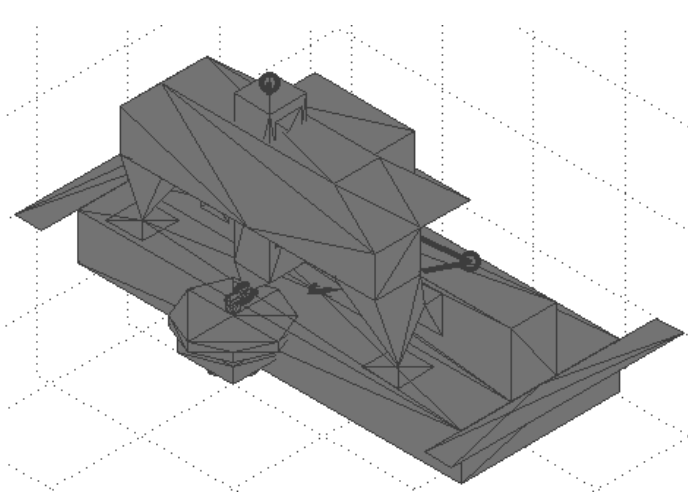

Figure 6.5: SimMechanics dynamic model 


\subsubsection{Frequency response}

A frequency response analysis of the model has shown that the first resonance occurs at $185 \mathrm{~Hz}$, which is a tilting mode of the $\psi$-axis. When this bearing is modified to a two sided suspension, the first resonance will be a radial resonance of the spindle with a $50 \mathrm{~kg}$ product at $280 \mathrm{~Hz}$.

\subsubsection{Typical time response}

In Figure 6.6, the typical time response of the model is shown. In this simulation, a $50 \mathrm{~kg}$ product was $0.5 \mathrm{~mm}$ off-centre at $1 \mathrm{rev} / \mathrm{s}$, the probe was vibrating at $6 \mathrm{~Hz}$ with $0.5 \mathrm{~mm}$ amplitude, and the floor vibrations were simulated with 0.1 $\mathrm{mm} / \mathrm{s}$ velocity steps at $15 \mathrm{~Hz}$. The first (a) shows the actual relative motion of the probe tip and the product. In a and bdirection, the $1 \mathrm{~Hz}$ spindle unbalance and the $6 \mathrm{~Hz}$ probe motion can be recognized. When this motion is corrected with the measurements from the metrology loop, the error vector components shown in the second (Figure 6.6b) remain. Again, the $1 \mathrm{~Hz}$ spindle and $6 \mathrm{~Hz}$ probe motion can be seen. In the magnification, the (negligible) influence of the floor vibrations can be seen as noise on the $6 \mathrm{~Hz}$ signal. When the resulting shape measurement error is calculated with these error vector components, a $6 \mathrm{~Hz}$ vibration with about $1.5 \mathrm{~nm}$ amplitude remains.
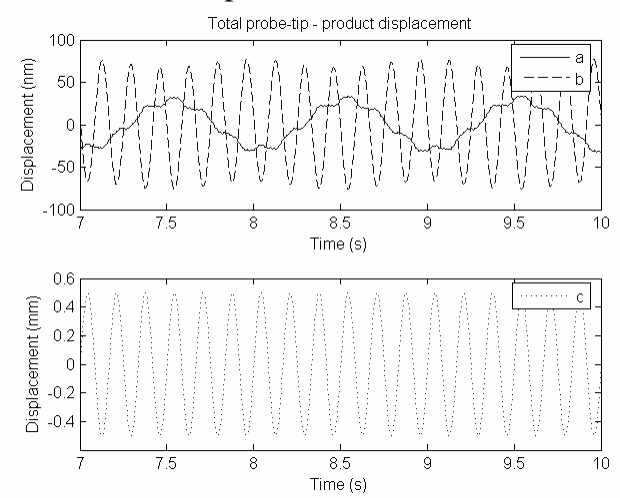

Figure 6.6a: Typical response

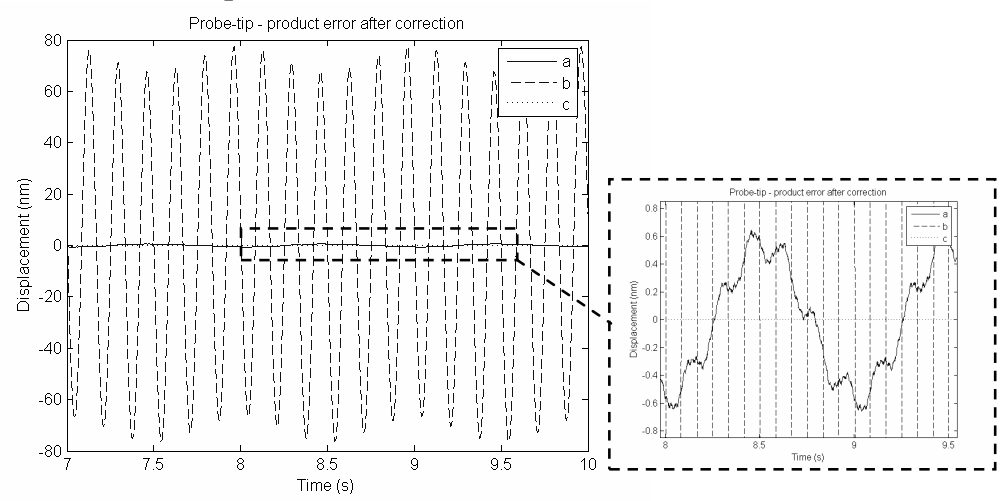

Figure 6.6b: Typical corrected response

\subsubsection{Floor vibrations}

Simulation of step shaped floor vibrations from $0.01 \mathrm{~mm} / \mathrm{s}$ (laboratory) $-0.1 \mathrm{~mm} / \mathrm{s}$ (workshop) between 5 and $15 \mathrm{~Hz}$ result in relative motion in a and c-direction of maximum $25 \mathrm{~nm}$. After correction with the measurements of the $\mathrm{R}$ and Z-interferometer, sub-nanometer errors remain. Because the lasers measure directly to the probe, floor vibrations thus probably cause negligible shape measurement uncertainty.

\subsubsection{Spindle unbalance}

From workshop experience, it may be assumed that a product can be aligned to within $0.1 \mathrm{~mm}$. This unbalance has been simulated for products up to $50 \mathrm{~kg}$ and spindle speeds up to $4 \mathrm{rev} / \mathrm{s}$. This results in relative errors up to respectively 30, 20 and $20 \mathrm{~nm}$ in a, b and c-direction. When corrected with the measurements of the metrology system, the remaining errors in a and c-direction are negligible. Since the b-direction is not measured, this 20 $\mathrm{nm}$ remains. Figure 6.7 shows the resulting shape measurement error per degree of local surface slope. As can be seen, this uncertainty is negligible compared with the static uncertainty calculated in 6.4.

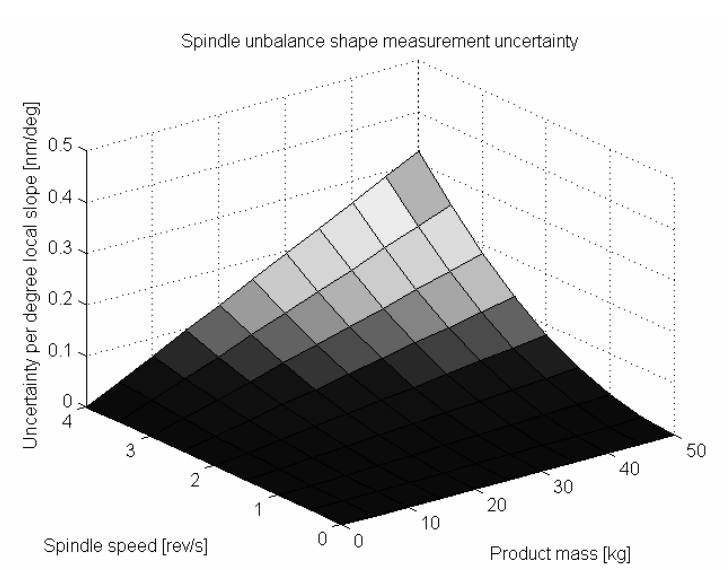

Figure 6.7: Spindle unbalance shape measurement uncertainty 


\subsubsection{Probe motion}

The errors caused by probe vibration are mainly caused by the reaction forces from accelerations of the probe tip, which cause the $\psi$-axis to tilt. This tilting causes the probe tip to move in b-direction (out of the measurement plane), up to $80 \mathrm{~nm}$ for $5 \mathrm{rad} / \mathrm{m}$ at $1.5 \mathrm{~m} / \mathrm{s}$. The resulting shape measurement uncertainty per degree of local surface slope is shown in Figure 6.8.

The required acceleration of the probe tip depends on the local curvature and local tangential speed of the surface. By adjusting the spindle speed, the magnitude of the error caused by the probe can thus be controlled. A better solution is however to change the $\psi$-axis bearing to a symmetrical setup around the probe, which cures this problem and probably reduces the probe induced dynamic error to a negligible amount.

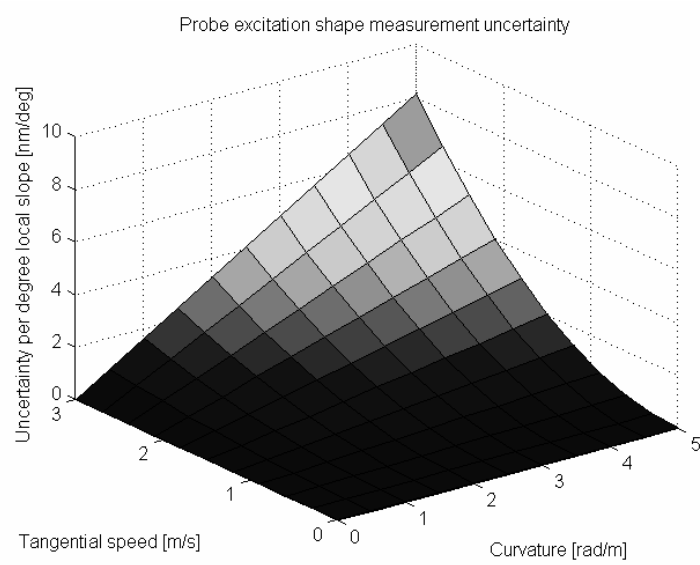

Figure 6.8: Probe motion shape measurement uncertainty

\subsection{Total shape measurement uncertainty estimation}

The total shape measurement uncertainty is the sum of the static and the dynamic uncertainties. Since floor vibrations and spindle unbalance cause negligible shape measurement uncertainty, this effectively means the sum of the static uncertainty plus the probe motion uncertainty. For some typical surfaces with diameter (D), number of waves (n), amplitude (a), spindle speed ( $\dot{\theta}$ ) and estimated measurement time (T) the expanded uncertainty (U) has been listed in Table 6.3. The measurement is based on a point spacing between 1 and $2 \mathrm{~mm}$ and 5 revolutions per track.

\begin{tabular}{|l|l|l|l|l|l|l|l|l|}
\hline Surface & $\mathbf{D}[\mathbf{m m}]$ & $\mathbf{n}[-/ \mathbf{r e v}]$ & $\mathbf{a}[\mathbf{m m}]$ & Slope $\left[^{\circ}\right]$ & $\mathbf{C u r v}[\mathbf{r a d} / \mathbf{m}]$ & $\dot{\theta}[\mathbf{r e v} / \mathbf{s}]$ & $\mathbf{T}[\mathbf{m i n}]$ & $\mathbf{U}[\mathbf{n m}]$ \\
\hline Aspheres & $50-500$ & - & - & - & - & $2-1$ & $2-15$ & $24-37$ \\
\hline Slightly free-form & 100 & 2 & 0.1 & 0.25 & 0.2 & 2 & 4 & 25 \\
\hline Medium free-form & 350 & 5 & 0.5 & 0.82 & 0.4 & 2 & 9 & 29 \\
\hline Heavily free-form & 500 & 8 & 2.5 & 4.6 & 2.6 & 1 & 15 & 85 \\
\hline
\end{tabular}

Table 6.3: Estimated shape measurement uncertainty for some typical free-form surfaces

Some final remarks on the above figures have to be noted:

- The estimated individual calibration uncertainties are still to be proven by a calibration design

- Temperature variations during a measurement have not yet been taken into account. The short measurement time certainly decreases the sensitivity to thermo-mechanical and environmental changes, but to what extent will be determined by the detailed design, the materials and the applied conditioning and shielding of the machine.

- The potentially positive effect of data processing (averaging, filtering, fitting) has not yet been taken into account.

Although the above figures may not be entirely accurate, they do indicate a significant improvement compared to conventional universal measurement techniques. Up to medium free-form surfaces $(\mathrm{a}=0.5 \mathrm{~mm})$ the $30 \mathrm{~nm}$ uncertainty goal may even be met. This proves the suitability of the proposed measurement machine design.

\section{CONCLUSION}

A new universal non-contact measurement machine design for measuring free-form optics with $30 \mathrm{~nm}$ expanded uncertainty has been presented. In the cylindrical machine concept, an optical probe with $5 \mathrm{~mm}$ range is positioned over the surface by a motion system. Due to the $2^{\text {nd }}$ order error effect when measuring smoothly curved surfaces, only 6 position measurement errors are critical (nanometer level). A separate metrology system directly measures these critical directions of the probe and the product relative to a metrology frame, canceling out most stage errors.

An uncertainty estimation has been performed for the presented design, including a calibration uncertainty estimation and a dynamic analysis. Machine dynamics certainly cause relative motion between probe and product, but due to the 
non-contact nature of the measurement and the short metrology loop, these motions do not cause significant measurement errors. The resulting shape measurement uncertainty for aspheres up to medium free-forms is between 24 and $37 \mathrm{~nm}$, and $30-90 \mathrm{~nm}$ for medium to heavily free-form surfaces. The suitability of the proposed design is herewith confirmed.

Currently, a non-contact probe concept is being developed, of which proof-of-principle tests will be conducted soon. The metrology loop, in particular the metrology frame and interferometry system will also be further designed. The airbearing spindle is soon to arrive at TU/e to be tested, after which the base and the stages will be further designed and constructed. This will finally result in a complete and tested machine prototype.

\section{ACKNOWLEDGEMENTS}

The NANOMEFOS project is partially funded by the IOP Precision Technology program of the Dutch Ministry of Economic affairs. The authors would further like to thank Pieter Kappelhof and Jacob Jan Korpershoek of TNO for their efforts to the project and Wim van Amstel of Wimoptik and Simon Oldfield and Keith Jackson of NPL for their valuable design reviews.

\section{REFERENCES}

1. I.J. Saunders, Fabrication and metrology of freeform aluminum mirrors for the SCUBA-2 instrument, Proc. of SPIE Optical Manufacturing \& Testing VI, 2005, to be published

2. H. van Brug, M. Dorrepaal, I. Saunders, Polishing robot based on FJP with in-situ monitoring system, Proc. of SPIE, Vol. 5180, 2004, p. 101 - 106

3. D. Malacara, Optical shop testing, $2^{\text {nd }}$ edition, Wiley-Interscience, ISBN 0-471-52232-5, 1992

4. J.H. Burge, J.C. Wyant, Use of Computer Generated Holograms for Testing Aspheric Surfaces, ASPE Winter topical meeting on Free-form Optics, 2004, p. 45 - 50

5. P.R. Dumas, et.al., Flexible polishing and metrology solutions for free-form optics, ASPE Winter topical meeting on Free-form Optics, 2004, p. $39-44$

6. K. Becker, E. Heynacher, M400 - A Co-ordinate measuring machine with $10 \mathrm{~nm}$ resolution, Proc. of SPIE, Vol. 802, 1987, p. 209 - 216

7. M.M.P.A. Vermeulen, High-Precision 3D coordinate measuring machine: Design and Prototype Development, PhD Thesis, TU Eindhoven, ISBN 90-386-2631-2, 1999

8. T.A.M. Ruijl, Ultra Precision Coordinate Measuring Machine: Design, Calibration and Error Compensation, $\mathrm{PhD}$ Thesis, TU Delft, ISBN 90-6464-287-7, 2001

9. D.S. Anderson, J.H. Burge, Swing-arm Profilometry of aspherics, Proc. of SPIE, Vol. 2536, 1995, p. 169 - 179

10. N.A.J van der Beek, et.al., 'Deflectometry on aspheric surfaces': A new approach and generic solution for measuring 'free-form' aspheres, Proc. of the $3^{\text {rd }}$ Euspen conference Eindhoven, 2002, p. 657 - 660

11. P.Z. Takacs, et.al., Improvements in the accuracy and the repeatability of long trace profiler measurements, Appl. Opt., Vol. 38, No. 25, Sept. 1999, 5468 - 5479

12. M. Schulz, I. Weingärtner, Free-form measurement with high lateral resolution, Proc. of the $3^{\text {rd }}$ Euspen conference Eindhoven, 2002, p. $621-624$

13. R. Henselmans, N. Rosielle, P. Kappelhof, NANOMEFOS - Nanometer Accuracy Non-contact Measurement of Free-form Optical Surfaces, ASPE Winter topical meeting on Free-form Optics, 2004, p. 97 - 101

14. Patent EP1519144, Free-form optical surface measuring apparatus and method, 2003

15. T. Kohno, et.al., High precision optical surface sensor, Applied Optics, Vol. 27, No. 1, p. 103 - 108

16. J. Tan, J. Zhang, Differential confocal optical system using gradient-index lenses, Opt. Eng. 42(10), 2003, p. 2868 2871

17. P. de Groot, Three-color laser-diode interferometer, Appl. Opt., Vol. 30, No. 25, Sept. 1991, p. 3612 - 3616 